\title{
Cross-Sectional Serosurvey of Companion Animals Housed with SARS-CoV-2-Infected Owners, Italy
}

Barbara Colitti, Luigi Bertolotti, Alessandro Mannelli, Gianmarco Ferrara, Andrea Vercelli, Andrea Grassi, Claudio Trentin, Saverio Paltrinieri, Chiara Nogarol, Nicola Decaro, Emiliana Brocchi, Sergio Rosati

We conducted a serologic survey among dogs and cats in Italy to detect antibodies against severe acute respiratory syndrome virus 2 (SARS-CoV-2). We found that SARS-CoV-2 seroprevalence was higher among cats $(16.2 \%)$ than dogs $(2.3 \%)$. In addition, seroprevalence was higher among animals living in close contact with SARS-CoV-2-positive owners.

\begin{abstract}
A fter emerging in Wuhan, China, in December 2019, coronavirus disease (COVID-19), caused by severe acute respiratory syndrome coronavirus 2 (SARS-CoV-2), rapidly became a serious threat to human health worldwide (1-3). Italy has experienced one of the highest rates of human deaths in the world (4).

Questions concerning the role of companion animals in the COVID-19 pandemic arose after a dog in Hong Kong reportedly tested positive for SARS-CoV-2 (5). In addition, the World Organisation for Animal Health defined COVID-19 as an emerging disease in animals and began promoting surveys on the prevalence of SARS-CoV-2 infections among animals (6). In this context, serologic tests are essential for rapid and accurate screening of animal populations.

Few studies have been conducted to clarify the effects domestic animals have in sustaining the SARSCoV-2 transmission cycle (5,7-9; Q. Zhang et al., unpub. data, https://www.biorxiv.org/content/10.110 1/2020.04.01.021196v1). Because Italy suffered high
\end{abstract}

Author affiliations: University of Turin, Turin, Italy (B. Colitti, L. Bertolotti, A. Mannelli, S. Rosati); University of Naples, Naples, Italy (G. Ferrara); Clinica veterinaria Città di Torino, Turin, Italy (A. Vercelli); I-Vet srl Laboratorio di Analisi Veterinarie, Flero, Italy (A. Grassi); AUSL Valle d'Aosta, Aosta, Italy (C. Trentin); University of Milan, Milan, Italy (S. Paltrinieri); IN3Diagnostic, Turin (C. Nogarol); University of Bari, Bari, Italy (N. Decaro); Istituto Zooprofilattico Sperimentale della Lombardia e dell'Emilia Romagna, Brescia, Italy (E. Brocchi)

DOI: https://doi.org/10.3201/eid2707.203314
COVID-19 incidence rates and the country has $>32$ million companion animals, health authorities were interested in examining virus transmission between humans and animals. We conducted a cross-sectional serologic survey among domestic dogs and cats in Italy to identify a possible association between SARSCoV-2 infection in humans and animals. We used serologic tests to detect specific antibodies from animals living in close contact with SARS-CoV-2-positive human patients.

\section{The Study}

Blood was collected from pets during routine activities performed by veterinary practitioners, who shared serum samples with us. Owners provided written consent for research purposes. We used 198 samples, 130 from dogs and 68 from cats, collected during the March-June 2020 COVID-19 epidemic in Italy and 100 serum samples, 65 from dogs and from 35 cats, collected in different regions of Italy before 2019 as prepandemic controls.

A recombinant antigen corresponding to the nucleocapsid $(\mathrm{N})$ protein of SARS-CoV-2 has been expressed in human embryonic kidney 293T cells, which have been used to develop Eradikit COVID19IgG (IN3diagnostic, https://www.in3diagnostic. com) a sensitive and specific ELISA to detect SARSCoV-2 antibodies in human serum samples. However, our initial attempts to validate the specificity of this ELISA on pet serum samples were unsuccessful. We switched the reaction from solid-phase to solution-phase kinetics using the same antigen and the specificity improved. Thus, we used a novel immunoassay, xMAP (Luminex Corp., https://www. luminexcorp.com), which is based on paramagnetic beads. We developed a flow cytometry-based system and applied it to serum samples from cats and dogs.

To define the test's specificity, we analyzed preepidemic samples and expressed results as the mean 


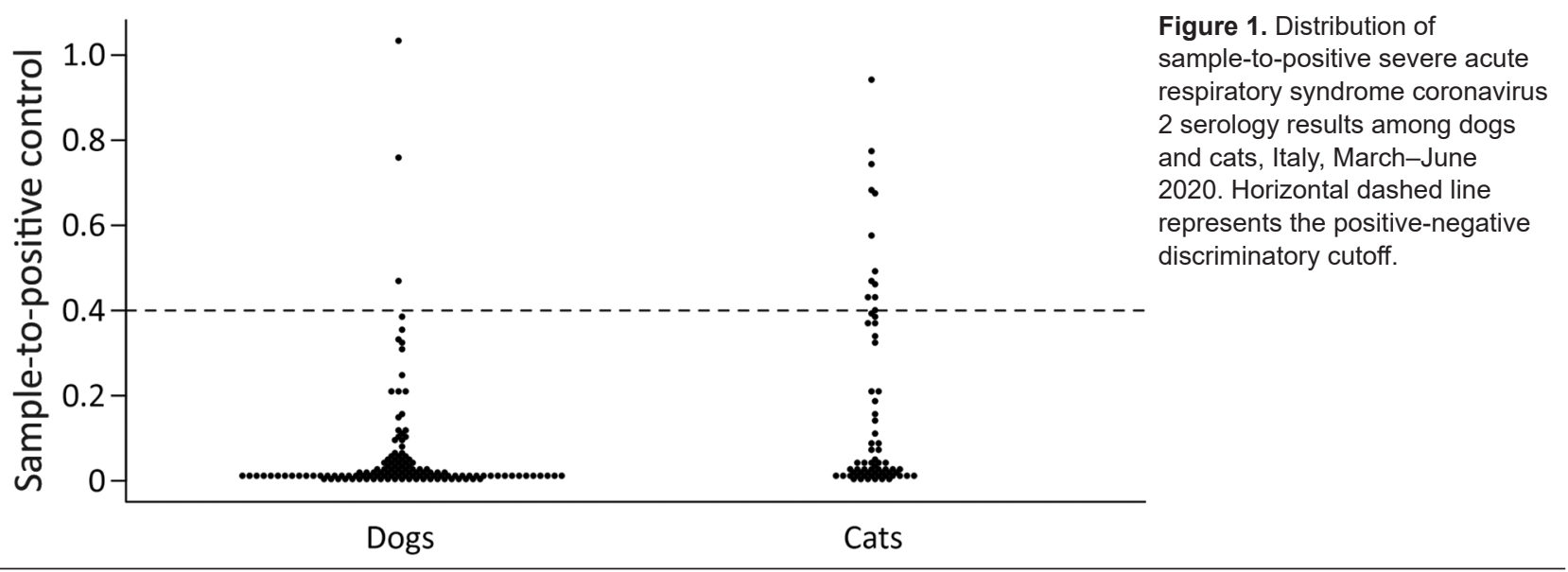

fluorescence intensity (MFI) ratio of a sample-to-positive control. On the basis of reactivity distribution, we set the discriminative cutoff to $40 \%$ MFI of the positive control. Using these specifications, we recorded diagnostic specificities of $96.5 \%$ (95\% CI 87.9\%-99.6\%) for dog serum and $100.0 \%$ (95\% CI $90.0 \%-100.0 \%)$ for cat serum.

Our choice of the viral $\mathrm{N}$ protein might raise concern because dogs and cats are susceptible to speciesspecific coronaviruses. The amino acid similarity between SARS-CoV-2 and the canine betacoronavirus, canine respiratory coronavirus, is slightly higher than canine and feline alphacoronaviruses (10), which

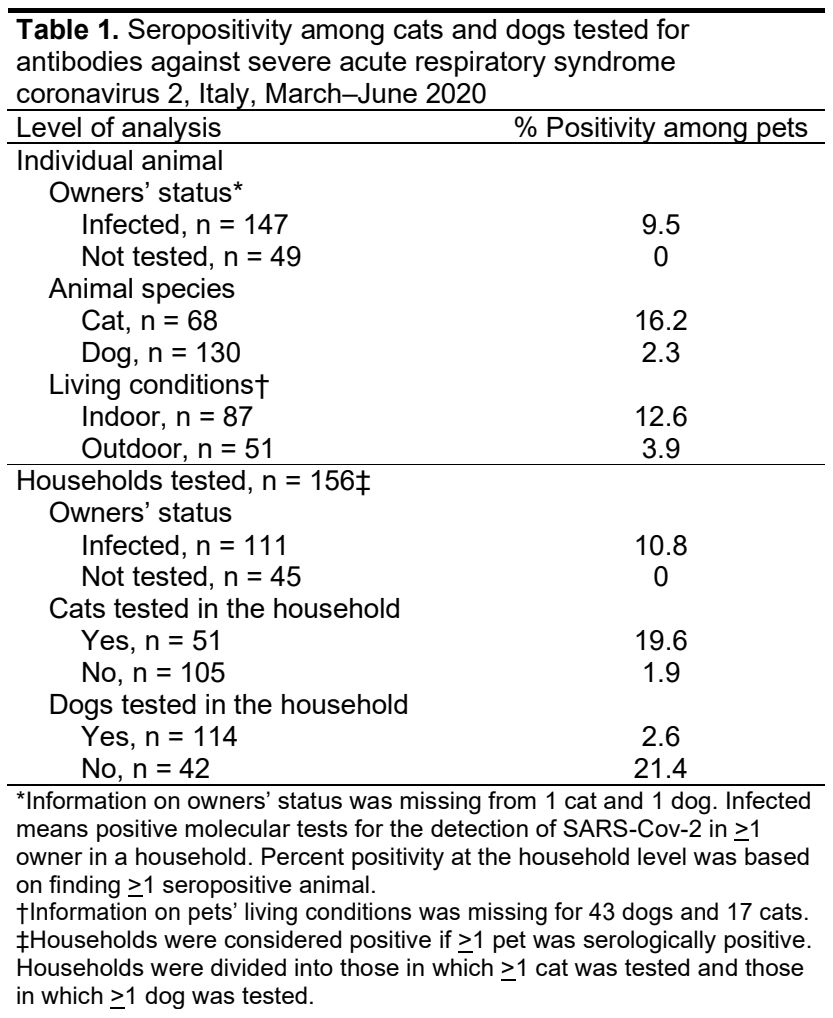

could explain the suboptimal specificity obtained in pre-epidemic dog samples. In fact, 2 serum samples gave reactivity slightly over the cutoff value. However, when potential cross-reactivity of the $\mathrm{N}$ protein between SARS-CoV-2 and endemic human coronaviruses was evaluated, no reactivity was shown against human coronaviruses 229E, OC43, HKU1, or NL63 by western blot or ELISA (11), suggesting that similar results might be expected from phylogenetically related feline and canine coronaviruses (12).

Among samples collected during the epidemic period, $7.1 \%(14 / 198)$ tested positive by the serologic test. In all, 147 animals (54 cats and 93 dogs) lived in households with SARS-CoV-2-positive owners. All 14 seropositive animals lived with SARS-CoV-2infected owners and percent positivity was greater among cats than dogs (Table 1; Figure 1). Among animals living with SARS-CoV-2-infected owners, $20.4 \%(11 / 54)$ of cats and $3.2 \%(3 / 93)$ of dogs were seropositive.

Exact logistic regression analysis indicated a positive association between owners' infections and seropositivity in individual animals, after adjusting for animal species (Figure 2 ). The odds of finding $\geq 1$ seropositive animal in a household were positively associated with owners' infection and with an increasing number of tested cats (Table 2; Appendix, https:/ / wwwnc.cdc.gov/EID/article/27/7/20-3314-App1. pdf). The association with owner infection was only statistically significant based on a 1-tailed hypothesis, whether the outcome was measured at the animal or household level.

Using exact logistic regression, we noted the percent of positive results was greater for animals living indoors only than for animals with access to the outside (odds ratio $3.4,95 \%$ CI $0.71-35.9$ ), but the association was not statistically significant $(p=0.15)$. Because information on living conditions was missing 


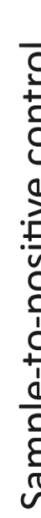
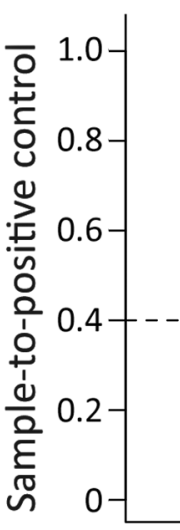
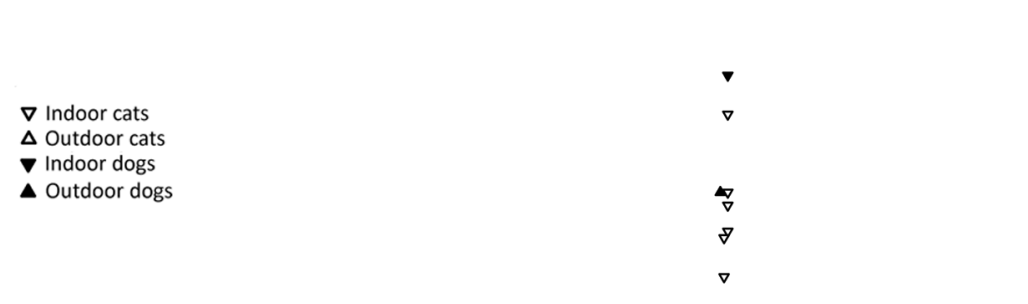

Figure 2. Distribution of sample-to-positive severe acute respiratory syndrome coronavirus 2 serology results among dogs and cats exposed and unexposed to positive owners, Italy, MarchJune 2020. Horizontal dashed line represents the positive-negative discriminatory cutoff. for 60 animals, we did not include this factor in the exact logistic regression analysis (Tables 1,2 ).

We found the proportion of serologic positivity increased with increasing length of exposure. We recorded the first SARS-CoV-2-positive animals 10 days after owners' diagnoses and all 14 seropositive cases were classified as positive after $\geq 54$ days of exposure (Appendix).

Among 5/14 positive animals, owners reported that their pets experienced clinical signs concurrent with the owner's COVID-19 illness. In particular, a 10-year-old male dog showed respiratory signs (cough, sneezing) after which he had vomiting and diarrhea in concomitance with the onset of the owners' symptoms; a 1-year-old dog showed mild respiratory signs characterized by cough and sneezing; a 12-year-old female cat showed respiratory signs characterized by rhinitis with abundant nasal discharge. Furthermore, a 13-year-old female cat was hospitalized for a brachial cephalic thrombosis and a 3-yearold male cat was hospitalized for interstitial pneumonia. Of note, 3 asymptomatic SARS-CoV-2-positive cats belonged to a single-family cluster in which both owners tested positive and hospitalized.

\section{Conclusions}

We detected antibodies against the SARS-CoV-2 N protein in pets living with SARS-CoV-2-infected owners. A higher percentage of feline samples tested positive, confirming a higher susceptibility and prevalence in cats than in dogs reported in previous experiments $(10,13)$. The susceptibility of cats to SARSrelated human coronaviruses also was reported in 2003 when a study confirmed that cats were susceptible to infection and could transmit the virus to other in-contact animals (14). The association between seropositivity in animals and the confirmed SARS-CoV-2 infection in $\geq 1$ of the animal's owners was statistically

Exposed

significant $(p<0.05)$ based on a 1-sided test assuming the owner's infection could not reasonably exert a protective effect on pets' infection.

We could not draw conclusions concerning the direction of viral transmission in this cross-sectional study. Nevertheless, our results, coupled with the direction of the association between seropositivity and length of exposure to an infected owner and living indoors, suggest that the development of antibodies in pets might be a consequence of viral transmission from their owners. Additional studies with more statistical power could confirm these relationships.

Based on our results, future studies should focus on overcoming test limitations by improving specificity in dog serum samples through detailed epitope mapping of the $\mathrm{N}$ protein. Additional studies also should examine routes and risk factors for transmission of SARS-CoV-2 from infected persons to susceptible pets and the potential role of pets in the COVID-19 pandemic. Clinical and pathological consequences of SARS-CoV-2 infection in cats and dogs also warrant further research.

In conclusion, our study on companion animals housed with SARS-CoV-2-infected humans confirms the susceptibility of domestic cats under natural exposure. Our data statistically support other findings that cats are more susceptible than dogs

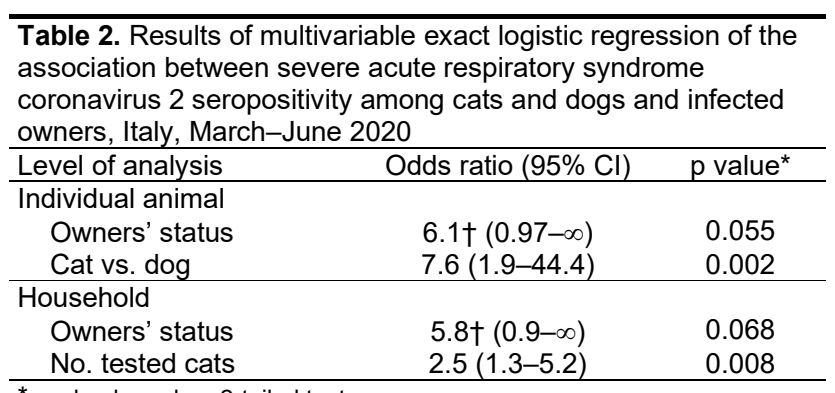

${ }^{*} \mathrm{p}$ value based on 2-tailed test.

†Median unbiased estimate. 
and that living in contact with $\geq 1$ SARS-CoV-2-infected person increases the risk for infection in pets. These results justify the need to adopt control measures in SARS-CoV-2-infected pet owners to reduce viral transmission to their companion animals.

\section{Acknowledgments}

We thank veterinary practitioners in Italy for their significant contributions from and help with sample collection. We also thank Alessandro Bellato for assisting with statistical analysis using Stata (StataCorp LLC, https:/ / www.stata.com).

The study was carried out in compliance with the national legislation with authorization by the Ministry of Health Legislative Decree 26/2014 (authorization no. 694/ 2020-PR). Blood samples were collected during routine activities performed by veterinary practitioners. Written consent was obtained from all owners for research purposes.

\section{About the Author}

Dr. Colitti holds a research grant position in the Department of Veterinary Sciences, University of Turin, Italy. Her primary research interest is the diagnosis of animal infectious diseases.

\section{References}

1. Zhou P, Yang XL, Wang XG, Hu B, Zhang L, Zhang W, et al. A pneumonia outbreak associated with a new coronavirus of probable bat origin. Nature. 2020;579:270-3. https://doi.org/10.1038/s41586-020-2012-7

2. Zheng J. SARS-CoV-2: an emerging coronavirus that causes a global threat. Int J Biol Sci. 2020;16:1678-85. https://doi.org/10.7150/ijbs.45053

3. Wu F, Zhao S, Yu B, Chen YM, Wang W, Song ZG, et al. A new coronavirus associated with human respiratory disease in China. Nature. 2020;579:265-9. https:/ / doi.org/ 10.1038/s41586-020-2008-3

4. World Health Organization. Coronavirus disease (COVID-19) dashboard 2020 [cited 2020 Nov 17]. https://covid19.who.int
5. Sit THC, Brackman CJ, Ip SM, Tam KWS, Law PYT, To EMW, et al. Infection of dogs with SARS-CoV-2. Nature. 2020;586:776-8. https:/ / doi.org/10.1038/ s41586-020-2334-5

6. World Organisation for Animal Health. Infection with SARS-Cov-2 in animals 2020; updated 2021 Jan [cited 2020 Nov 8]. https:/ / www.oie.int/app/uploads/2021/03/ en-factsheet-sars-cov-2.pdf

7. Shi J, Wen Z, Zhong G, Yang H, Wang C, Huang B, et al. Susceptibility of ferrets, cats, dogs, and other domesticated animals to SARS-coronavirus 2. Science. 2020;368:1016-20. https://doi.org/10.1126/science.abb7015

8. Halfmann PJ, Hatta M, Chiba S, Maemura T, Fan S, Takeda M, et al. Transmission of SARS-CoV-2 in domestic cats. N Engl J Med. 2020;383:592-4. https:/ / doi.org/10.1056/ NEJMc2013400

9. Bosco-Lauth AM, Hartwig AE, Porter SM, Gordy PW, Nehring M, Byas AD, et al. Experimental infection of domestic dogs and cats with SARS-CoV-2: Pathogenesis, transmission, and response to reexposure in cats. Proc Natl Acad Sci U S A. 2020;117:26382-8. https:/ / doi.org/10.1073/ pnas. 2013102117

10. Sharun K, Sircar S, Malik YS, Singh RK, Dhama K. How close is SARS-CoV-2 to canine and feline coronaviruses? J Small Anim Pract. 2020;61:523-6. https:/ / doi.org/10.1111/ jsap.13207

11. Guo L, Ren L, Yang S, Xiao M, Chang D, Yang F, et al. Profiling early humoral response to diagnose novel coronavirus disease (COVID-19). Clin Infect Dis. 2020;71:77885. https://doi.org/10.1093/cid/ciaa310

12. Lv H, Wu NC, Tsang OT-Y, Yuan M, Perera RAPM, Leung WS, et al. Cross-reactive antibody response between SARS-CoV-2 and SARS-CoV infections. Cell Rep. 2020;31:107725. PubMed https://doi.org/10.1016/ j.celrep.2020.107725

13. Salajegheh Tazerji $S$, Magalhães Duarte $P$, Rahimi $P$, Shahabinejad F, Dhakal S, Singh Malik Y, et al. Transmission of severe acute respiratory syndrome coronavirus 2 (SARS-CoV-2) to animals: an updated review. J Transl Med. 2020;18:358. PubMed https://doi.org/0.1186/ s12967-020-02534-2

14. Martina BE, Haagmans BL, Kuiken T, Fouchier RA, Rimmelzwaan GF, Van Amerongen G, et al. Virology: SARS virus infection of cats and ferrets. Nature. 2003;425:915. https://doi.org/10.1038/425915a

Address for correspondence: Barbara Colitti, University of Turin, Largo Paolo Braccini 2, 10095 Grugliasco, Turin, Italy; email: barbara.colitti@unito.it 